\title{
Acquisition of habitual visual attention and transfer to related tasks
}

\author{
Nikita A. Salovich ${ }^{1} \cdot$ Roger W. Remington ${ }^{1} \cdot$ Yuhong V. Jiang ${ }^{1}$
}

Published online: 11 July 2017

(C) Psychonomic Society, Inc. 2017

\begin{abstract}
Extensive research has shown that statistical learning affects perception, attention, and action control; however, few studies have directly linked statistical learning with the formation of habits. Evidence that learning can induce a search habit has come from location probability learning, in which people prioritize locations frequently attended to in the past. Here, using an alternating training-testing procedure, we demonstrated that the initial attentional bias arises from shortterm intertrial priming, whereas probability learning takes longer to emerge, first reaching significance in covert orienting (measured by reaction times) after about 48 training trials, and in overt orienting (measured by eye movements) after about 96 training trials. We further showed that location probability learning is persistent after training is discontinued, by transferring from a letter search task to a scene search task-emulating another characteristic feature of habits. By identifying the onset of probability learning and investigating its task specificity, this study provides evidence that probability cuing can induce habitual spatial attention.
\end{abstract}

Keywords Habitual attention · Statistical learning ·

Probability cuing $\cdot$ Visual search $\cdot$ Eyetracking

Habits play a central role in perception, attention, and action. As we become skilled, formerly deliberate components become habits (Graybiel, 2008; Wood \& Rünger, 2016). Current research on how repetition shapes learning has proceeded in parallel tracks. Using primarily animal models,

Nikita A. Salovich

salov013@umn.edu

1 Department of Psychology, University of Minnesota, S504 Elliott Hall, Minneapolis, MN 55455, USA one line of research has shown that repeatedly engaging in activities induces habits. A second line has examined statistical learning: the ability to act on the basis of consistent statistical regularities in the environment. These two lines of research have rarely intersected - studies on habits emphasize the repeated nature of behavior, whereas studies of statistical learning emphasize learning environmental regularities. In the present study, we investigated whether the attentional bias formed via statistical learning holds properties characteristic of habits, and therefore can be understood as habitual attention.

Habits are typically (1) implicit, (2) gradually acquired or learned, and (3) fixed or persistent patterns of behavior (Graybiel, 2008; Seger \& Spiering, 2011). They differ from biases, which need not be learned or persistent. Studies using location probability cuing have suggested that statistical learning elicits the formation of habitual spatial attention. When asked to search for a letter $\mathrm{T}$ among letter Ls, participants initially show no locational preference. However, if the $\mathrm{T}$ is found more often in one region, they become faster at locating the $\mathrm{T}$ in the high-probability region (Druker \& Anderson, 2010; Geng \& Behrmann, 2002). Like other forms of statistical learning (Chun \& Jiang, 1998), probability cuing reflects one's ability to extract environmental statistics and use this information to guide attention. Like other habits, probability cuing is implicit, persists for several days after acquisition, manifests a viewer-centered representation of space, and is not disrupted by secondary working memory load (Jiang, 2017). Thus, statistical learning not only affects one's internal representation of the world but may also lead to habit formation.

However, it remains unclear whether location probability learning is acquired gradually like other habits (Graybiel, 2008; Seger \& Spiering, 2011). Whereas most habits take many repetitions to form, the search advantage in high- 
probability locations emerges rapidly, sometimes becoming significant after a dozen trials (Won, Lee, \& Jiang, 2015). Given this early onset, some researchers have questioned whether participants acquire any statistical learning. Walthew and Gilchrist (2006) proposed that the search advantage reflects short-term intertrial priming (Kristjánsson \& Campana, 2010). When the target appears more often in some locations, it also repeats its location more often there than elsewhere; therefore, repetition priming rather than statistical learning may drive the apparently early onset of the attentional bias. To determine when probability learning occurred, previous research had taken a pseudorandom approach in which the target's location was not allowed to repeat on consecutive trials (Jones \& Kaschak, 2012; Kabata \& Matsumoto, 2012; Walthew \& Gilchrist, 2006). However, this method introduces new statistical regularities that counter probability learning (for a discussion, see Druker \& Anderson, 2010). A different approach would be needed to determine how much training is needed to acquire probability learning.

In this study we adopted a simpler experimental approach to identify the onset of probability cuing. It is modified from a previous two-phase design involving blocks of "biased" and "unbiased" trials (Jiang, Swallow, Rosenbaum, \& Herzig, 2013). In the earlier paradigm, the target is found more often in one quadrant than in others during a "biased" training phase. In a subsequent "unbiased" testing phase, the target was equally likely to appear in all quadrants. Because shortterm repetition priming is not confounded with statistical learning in the unbiased phase, this phase can unambiguously index statistical learning. Unfortunately, the use of a long training phase, typically lasting several hundred trials, does not permit an assessment of when statistical learning emerges. Here we aimed to more accurately identify the onset time of probability cuing. We interleaved short blocks of biased with unbiased trials in order to probe statistical learning at frequent intervals during the experiment.

A second goal of this study was to examine probability cuing in terms of another characteristic feature of habits: fixedness, or long-term persistence. Most habits, once acquired, persist even when the behavior no longer achieves desirable outcomes. This persistence shows some, but not complete, task specificity, since it may persist in related tasks. For example, Bayley, Frascino, and Squire (2005) sequentially presented amnesic patients with eight pairs of objects and had the patients guess which object was correct. Over multiple sessions, the patients learned to choose the correct object from the pair despite having no explicit memory. The habit of identifying the correct object did not transfer when all objects were presented for patients to sort into correct and incorrect piles. However, it did transfer from a manual task (flipping the object over) to a verbal task (stating "left" or "right" as the correct object). These findings show that habits are likely to exhibit moderate degrees of transfer to related tasks. Thus, the present study tested whether probability cuing acquired from a letter search task persists and transfers to a scene search task, as would be expected if it were a habit.

\section{Method}

\section{Participants}

Forty college students ( 25 females, 15 males; $18-26$ years old) completed this study. All had normal or corrected-to-normal visual acuity.

\section{Equipment}

The participants were tested individually. Stimuli were generated using the Psychophysics Toolbox (Brainard, 1997; Pelli, 1997) implemented in MATLAB (www.mathworks.com) and displayed on a 17 -in. CRT monitor $(1,024 \times 768$ pixels; $75 \mathrm{~Hz}$ ). All participants contributed to the behavioral data, and 20 were also tested with eyetracking. Viewing distance was constrained to $90 \mathrm{~cm}$ for the eyetracked participants and was unconstrained for those without eyetracking.

An EyeLink 1000 tracked 20 participants' left eyes at a sampling rate of $1000 \mathrm{~Hz}$. Eye position was calibrated using a nine-point calibration. Eye position was verified before each trial and recalibrated, if necessary.

\section{Stimuli}

The T-among-Ls search task displayed 12 randomly located items on an invisible $10 \times 10$ grid $\left(16^{\circ} \times 16^{\circ}\right)$. The items included one $\mathrm{T}$ (target) and $11 \mathrm{Ls}$ (distractors; $1^{\circ} \times 1^{\circ}$ ). The stimuli were white against a black background (Fig. 1B). All items were rotated in four random orientations $\left(0^{\circ}, 90^{\circ}, 180^{\circ}\right.$, or $270^{\circ}$ ). The location of the target was manipulated to vary in the frequency it appeared in each quadrant (Fig. 1A). There were ten blocks of the T-among-Ls task, each containing 36 trials. In the first 12 trials of each block, the T appeared in all quadrants equally (three trials per quadrant). These comprised the unbiased trials. In the last 24 trials, the $T$ appeared in a high-probability "rich" quadrant $50 \%$ of the time, and in each of the three low-probability "sparse" quadrants $16.7 \%$ of the time. These were the biased trials. The high-probability quadrant was randomly chosen and counterbalanced across participants.

Following completion of the T-among-Ls blocks, participants performed a scene search task. This task displayed one yellow arrow (RGB values: [160 160 0]; size $0.6^{\circ} \times 0.2^{\circ}$ ) on a photograph $\left(780 \times 780\right.$ pixels; $16^{\circ} \times 16^{\circ}$; Fig. 2$)$. All 12 possible photographs were of natural scenes that included a road. In each scene's upright orientation, the top region was typically sky, which was visually simpler than the bottom 
a

$\begin{aligned} & \text { Unbiased Distribution } \\
& (12 \text { trials })\end{aligned}$
\begin{tabular}{|c|c|c|c|c|}
\hline $25 \%$ & $25 \%$ & & $\begin{array}{c}\text { Biased Distribution } \\
(24 \text { trials })\end{array}$ \\
\hline $25 \%$ & $25 \%$ \\
\hline & $\begin{array}{r}50 \% \\
\text { Rich Q }\end{array}$ & $\begin{array}{c}17 \% \\
\text { Sparse Q }\end{array}$ \\
\hline $\begin{array}{c}17 \% \\
\text { Sparse Q }\end{array}$ & $\begin{array}{c}17 \% \\
\text { Sparse Q }\end{array}$ \\
\hline
\end{tabular}

b

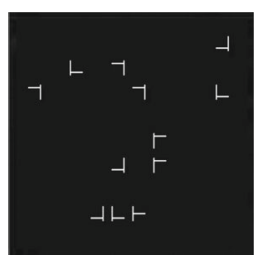

Fig. 1 Pictorial representations of the design and stimuli used in the Tand-L letter search task. (A) It was equally probable for the target " $T$ " to appear in all quadrants in Trials 1-12, but it became more probable for the target to appear in a "rich," high-probability quadrant in Trials 13-36. This alternation occurred ten times. (B) Image of a T-and-L task trial

region and made segmenting the target from the top easier. To ensure equivalent search difficulty across quadrants, scenes were presented in the original as well as in reflected, inverted, and inverted-reflected orientations. The scene and its orientation were randomly selected for each trial. The arrow was randomly rotated in four orientations $\left(0^{\circ}, 90^{\circ}, 180^{\circ}\right.$, or $270^{\circ}$ ), and its location was randomly selected on each trial.

\section{Procedure}

The experimenter administered practice trials (24 T-among-Ls trials and 12 scene trials). Participants were not informed of the target's location probability. Following practice, the participants completed 360 trials of the T-among-Ls task and 120 trials of the scene search task.

To initiate each trial, the eyetracked participants fixated a center dot and pressed the spacebar, and the search display was presented when the central fixation was successful. Those without eyetracking clicked on a red $\operatorname{dot}\left(0.2^{\circ} \times 0.2^{\circ}\right)$ to center their fixation. The display of either the T-among-Ls or the scene search task (depending on the block) appeared until a response had been made or $10 \mathrm{~s}$ had elapsed. Participants reported the target's orientation by pressing one of four keys as quickly and accurately as possible. Rising tones followed correct responses; a low buzz followed incorrect responses. If the target was not found in $10 \mathrm{~s}$, the display froze, a voice told the participants that they had timed out, and a red circle indicated the location of the target.

a

\begin{tabular}{|l|l|}
\hline $25 \%$ & $25 \%$ \\
\hline $25 \%$ & $25 \%$ \\
\hline
\end{tabular}

b
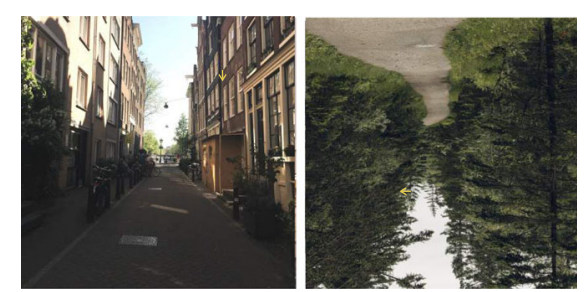

Fig. 2 Pictorial representations of the design and stimuli used in the scene search task. (A) It was equally probable for the target (arrow) to appear in each of the four quadrants in all trials. (B) Two images of possible trials

\section{Recognition task}

At the conclusion of the experiment, explicit awareness was assessed by asking participants whether they believed the $\mathrm{T}$ had appeared more often in a particular quadrant. Regardless of their answer, participants were told that the $\mathrm{T}$ had been located more often in one quadrant, and they were asked to identify that quadrant. Participants then reported their level of confidence using a 4-point scale (1 completely guessing, 2 somewhat confident, 3 almost certain, 4 completely certain).

\section{Results}

\section{The letter search task}

\section{$T / L$ search accuracy}

Participants rarely timed out (four of 14,400 trials). Their overall accuracy approached 99\% (Table 1). An analysis of variance (ANOVA) on block type (biased or unbiased) and target quadrant (high or low probability) showed that accuracy was not significantly affected by either variable or by their interaction, $F \mathrm{~s}<1$. Incorrect trials were excluded from the reaction time (RT) analysis.

\section{$T / L$ search $R T$}

Unbiased trials Each block began with 12 unbiased trials, wherein the target appeared equally across quadrants (Fig. 3, left). An ANOVA on target quadrant and block showed a significant main effect of the target's quadrant, $F(1,39)=$ 25.43, $p<.001, \eta_{\mathrm{p}}{ }^{2}=.40$. Participants were faster in the high-probability quadrant (defined by the biased trials), even though in the unbiased trials the target was equally probable across quadrants. The main effect of block was significant, indicating that RTs became faster as the experiment progressed, $F(9,351)=18.80, p<.001, \eta_{\mathrm{p}}{ }^{2}=.33$. We found a significant interaction between target quadrant and block, $F(9,351)=2.15, p<.03, \eta_{\mathrm{p}}{ }^{2}=.05$, accompanied by a significant linear trend and a quadratic trend in the interaction term, $p \mathrm{~s}<.05$. The RT advantage in the high-probability quadrant increased with training, and this increase was greater early during training than later.

RTs were comparable between the high- and lowprobability conditions in Block 1, prior to any exposure to location probability training. The earliest block in which RTs diverged between these conditions was Block $3, t(39)=3.23$, $p<.003$. This occurred after two blocks, or 48 biased trials.

Biased trials Biased trials were administered after the 12 unbiased trials within each block (Fig. 3, right). An ANOVA on the target's quadrant (high or low probability) and block (1- 
Table 1 Mean accuracies in the letter search and scene search tasks

\begin{tabular}{llll}
\hline Target Quadrant & T/L Unbiased Blocks & T/L Biased Blocks & Scene Search \\
\hline Low probability & $98.7 \%(0.2 \%)$ & $98.8 \%(0.2 \%)$ & $97.3 \%(0.5 \%)$ \\
High probability & $98.7 \%(0.4 \%)$ & $98.8 \%(0.2 \%)$ & $97.3 \%(0.6 \%)$ \\
\hline
\end{tabular}

Standard errors of the means are shown in parentheses

10) showed that participants were faster finding the target in the high-probability quadrant, $F(1,39)=121.70, p<.001, \eta_{\mathrm{p}}{ }^{2}$ $=.76$, and in later than in earlier blocks, $F(9,351)=26.56, p<$ $.001, \eta_{\mathrm{p}}{ }^{2}=.41$. Probability cuing did not increase significantly in later blocks, $F<1$ for the interaction between target quadrant and block. The RT advantage in the high-probability quadrant appeared early: It was already significant in Block $1, t(39)=2.11, p<.05$, yet was not sustained in the subsequent block of unbiased trials (i.e., Block 2 of the unbiased trials).

\section{$T / L$ search eye movement}

It is possible that probability cuing does not reflect a learned bias of attention, but instead decision-related effects (Kunar, Flusberg, Horowitz, \& Wolfe, 2007). This ambiguity arises because the RT is an aggregate measure of all processes in a trial, including both early and late effects. Previous studies have used first saccadic eye movements to index early attentional allocation (e.g., Jiang, Won, \& Swallow, 2014; Peterson $\&$ Kramer, 2001). The mean latency for the first saccadic eye movement following trial onset was about $165 \mathrm{~ms}$ (Table 2). An ANOVA on block type (unbiased or biased) and target quadrant (high or low probability) showed that the saccade latency was unaffected by either factor or their interaction, $F$ $<1$. Thus, the first saccade was initiated rapidly. Because the latency was insensitive to target location, the first saccades were likely made before participants had accrued information about the target's location on the current trial. The direction of the first saccade, therefore, reflects an attentional bias acquired from previous trials.

Averaged across blocks, participants directed the first saccade toward the high-probability quadrant significantly more often than chance $(25 \%)$ in both unbiased blocks $(35 \%), t(19)$ $=2.17, p=.043$, and biased blocks $(47 \%), t(19)=5.73, p<$ .001 (Fig. 4).

To evaluate how the tendency to saccade toward the highprobability quadrant changed over time, we performed an ANOVA on block type (biased or unbiased) and block number (1-10). We found a significant main effect of block type, $F(1$, 19) $=50.45, p<.001, \eta_{\mathrm{p}}{ }^{2}=.73$, in which participants were more likely to direct the first saccade toward the highprobability quadrant in biased than in unbiased blocks. The first-saccade bias toward the high-probability quadrant became stronger with training, manifested as a significant effect of block number, $F(9,171)=8.37, p<.001, \eta_{\mathrm{p}}{ }^{2}=.31$, accompanied by a significant linear trend, $F(1,19)=22.85, p<$ $.001, \eta_{\mathrm{p}}{ }^{2}=.55$. No interaction between the variables was found, $F<1$. Thus, even during unbiased trials, participants were increasingly likely to saccade toward the highprobability quadrant as training progressed, demonstrating the gradual onset of the attentional bias. The earliest point in the unbiased trials at which the first saccade became significantly biased toward the high-probability quadrant was Block $5, t(19)=2.58, p<.02$, consistent with the RT data, demonstrating that participants acquired a preference for the highprobability quadrant only after several blocks of training.

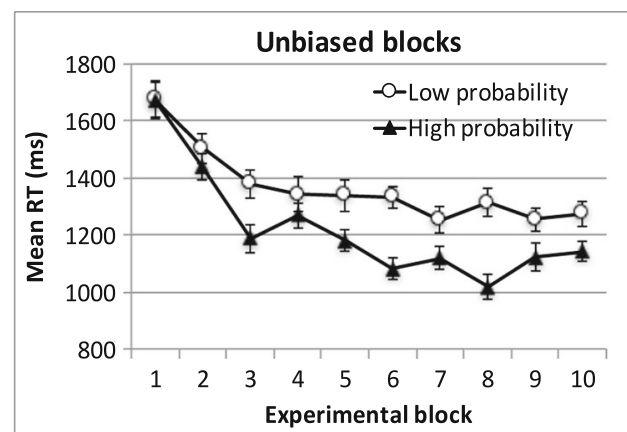

Fig. 3 Results of the T-among-Ls search task, depicting the average reaction times (RTs) for locating the target stimulus in the highprobability quadrant and the low-probability quadrants across blocks.

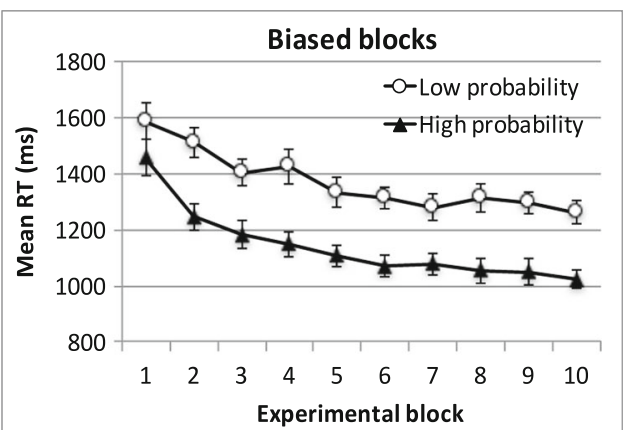

Unbiased block $=$ first 12 trials of each T-among-Ls search block (left). Biased block $=$ last 24 trials of each T-among-Ls search block (right). Error bars show \pm 1 standard error of the mean 
Table 2 Mean latencies of the first saccadic eye movement in the letter search and scene search tasks (in milliseconds)

\begin{tabular}{llll}
\hline Target Quadrant & T/L Unbiased Blocks & T/L Biased Blocks & Scene Search \\
\hline Low probability & $165(5)$ & $166(4)$ & $184(12)$ \\
High probability & $168(6)$ & $166(5)$ & $182(13)$ \\
\hline
\end{tabular}

Standard errors of the means are shown in parentheses

\section{The scene search task}

Next we examined whether the probability cuing acquired from the T-among-Ls search task transferred to the scene search task.

\section{Scene search accuracy}

Participants timed out on approximately $2 \%$ of the scene search trials. The time-out rates were equivalent in the (Tamong-Ls task's) high-probability (2.1\%) and lowprobability $(2.2 \%)$ quadrants, $p>.50$. Incorrect responses were rare, approximately $2.7 \%$ (Table 1 ), and were comparable between the high- and low-probability quadrants, $t(39)<$ 1. Only correct trials were included in the RT analysis.

\section{Scene search $R T$}

The scene search task included 120 trials; the target arrow was equally probable to appear in all quadrants. To index a change in performance over time while retaining enough trials per cell, we divided the scene search task into the first and last 60 trials (Fig. 5). The previously learned attentional preference for the high-probability quadrant persisted in the scene search task in RTs (Fig. 5, left). An ANOVA comparing RTs for the

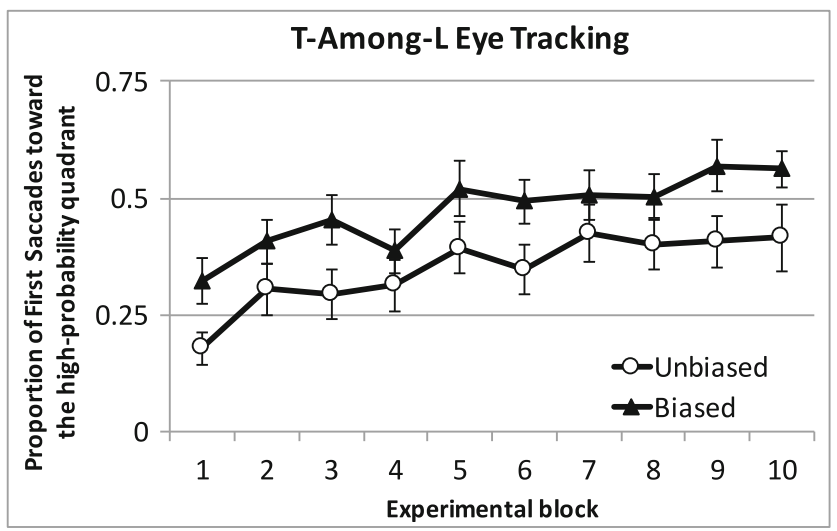

Fig. 4 Eyetracking results of the T-among-Ls search task, depicting the proportions of first saccades that were directed toward the highprobability quadrant in the biased and unbiased trials of the task. Chance level is .25 . Each experimental block included 12 unbiased trials followed by 24 biased trials. Error bars show \pm 1 standard error of the mean target's quadrant (high or low probability in the preceding Tamong-Ls task) and experiment half revealed significant main effects of target quadrant, $F(1,39)=4.19, p<.05, \eta_{\mathrm{p}}{ }^{2}=.10$, and experimental half, $F(1,39)=11.97, p<.001, \eta_{\mathrm{p}}{ }^{2}=.24$. The two variables did not interact, suggesting that the RT advantage in the high-probability quadrant was maintained in both experimental halves, $F<1$.

\section{Scene search eye movement}

The mean saccade latency in the scene search task was under $200 \mathrm{~ms}$ (Table 2) and was unaffected by the target's quadrant, $t(19)=0.51, p>.50$. Aggregated across all scene search trials, participants directed their first saccade toward the high-probability quadrant on $32 \%$ of the trials-marginally higher than we would expect by chance, $t(19)=2.08, p=.051$. Depicted in Fig. 5 (right), the saccade effect was stronger in the first than in the second half. In the first half, participants directed $34 \%$ of first saccades toward the high-probability quadrant, significantly above chance, $t(19)=2.84, p<.01$. This declined to a nonsignificant effect of $29 \%$ in the second half, $t(19)=1.22, p>.20$. The decline from the first to second half of the scene task was significant, $F(1,19)=6.25, p<.025$.

\section{Explicit recognition}

Fifteen of the 40 participants correctly identified the highprobability quadrant in the forced choice recognition task. This proportion was not significantly above chance, $\chi^{2}(1)=$ $3.33, p>.05$. Among those 15 participants, eight had initially reported the target's location to be random. Thus, only seven of the 40 participants reported being aware of the target's biased distribution. The median reported confidence ratings did not differ between aware and unaware participants (2 somewhat confident). To examine possible associations between awareness and probability learning, we calculated an index of RT saving, computed as the difference in RTs between the low- and high-probability quadrants divided by the mean RT (Table 3). RT savings were similar for aware and unaware participants in all phases of the experiment, $F \mathrm{~S}$ $<1$ when comparing aware and unaware participants. Location probability learning was thus largely implicit. 


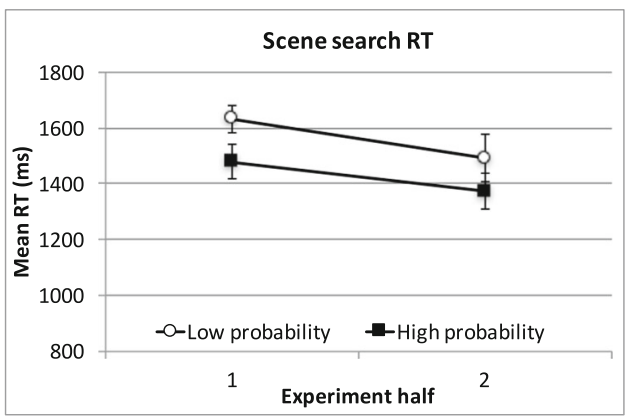

Fig. 5 (Left) Results of the scene search task, depicting the average reaction times (RTs) for locating the target stimulus (arrow) in the highprobability quadrant and the low-probability quadrants. Each half of the

\section{Discussion}

This study addressed two questions about location probability learning: the amount of training needed to acquire a durable attentional bias and the degrees to which the bias persists and transfers to a related task. To measure the cumulative effect of training and to probe the gradual onset of learning, we interleaved short segments of unbiased and biased trials in the Tamong-Ls search task. A significant RT advantage toward the high-probability quadrant emerged in the first biased block, but this effect did not reach significance until the third unbiased block. Previous studies using the two-phase design, wherein a long training phase was followed by a testing phase, have reported significant probability cuing in the first training (biased) block (e.g., Won et al., 2015). However, this RT advantage most likely reflects location repetition priming (Walthew \& Gilchrist, 2006). Consistent with Kabata and Matsumoto (2012), probability learning onsets gradually with training, resembling the temporal onset of habits.

Similarly, several blocks of training were needed before participants acquired a tendency to saccade toward the highprobability quadrant. The stronger saccadic effect for biased than for unbiased trials suggests that short-term repetition effects were also present. The first-saccade effect strengthened as the experiment progressed, becoming significant in the fifth unbiased block, after approximately 96 trials of training. The later onset in first saccades than in RTs may reflect a difference in measurement sensitivity. If probability learning affects not just the first but also subsequent saccades, the first-saccade

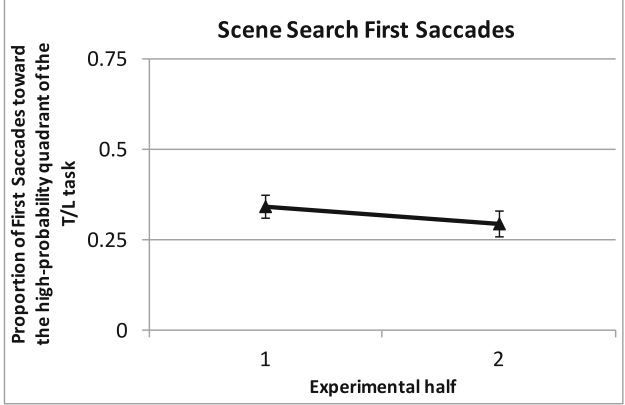

experiment contained 60 trials. (Right) Proportions of first saccadic eye movements toward the high-probability quadrant. Chance level is .25 . Error bars show \pm 1 standard error of the mean

data would provide only a partial sample of learning. Alternatively, overt orienting may take more repetitions than covert orienting to become habitual.

In the biased blocks, participants directed their first saccades $47 \%$ of the time toward the high-probability quadrant, demonstrating probability matching to the target's location probability (Geng \& Behrmann, 2002). However, the persisting attentional bias in the unbiased blocks violated probability matching. Repetition priming may initially induce a context-specific strategy of biased attending that resembles probability matching, yet the behavior becomes habitual with robust training. Once formed, the habit persists in novel contexts, in violation of probability matching.

The finding that gradual training is needed supports the idea that location probability cuing induces a change in search habits. The training produced in subsequent blocks constitutes overtraining, or additional training after the initial onset of the learned bias. Overtraining may be necessary for the formation of robust habits and for the spatial bias to become fully automatic (Yin \& Knowlton, 2006). Because our study was designed to probe the initial onset, it does not address how overtraining affects the robustness of probability cuing - a topic of interest for future research.

The transfer data show that location probability learning persists and transfers to related yet distinct tasks. Both the Tamong-Ls and the scene search tasks involve visual search; however, whereas the T-among-Ls task requires discrimination among already-segmented stimuli, the scene search places high demands on perceptually segmenting the target

Table 3 Reaction time (RT) savings for participants who became aware of the target's location probability and for those who remained unaware

\begin{tabular}{llll}
\hline & T/L Unbiased Blocks & T/L Biased Blocks & Scene Search \\
\hline Unaware $(N=33)$ & $10 \%(2 \%)$ & $18 \%(2 \%)$ & $8 \%(5 \%)$ \\
Aware $(N=7)$ & $11 \%(4 \%)$ & $19 \%(2 \%)$ & $13 \%(9 \%)$
\end{tabular}

RT saving was the RT difference between the low- and high-probability quadrants divided by the mean RT. Standard errors of the means are shown in parentheses 
from the background. The significant transfer between tasks suggests that the learned attentional bias is not entirely stimulus-specific, resembling a habit in its persistence. Previous studies showed that frequently moving one's eyes to a quadrant is neither necessary nor sufficient for location probability learning (Jiang, 2017). Therefore, probability cuing may be considered a form of attentional habit rather than a motor habit.

The transfer between the T-among-Ls and scene search tasks is an example of near transfer (i.e., transfer across closely related tasks), since both tasks entail serially scanning the display to find a target. Therefore, our finding should not be taken as evidence that participants have become generally more efficient at spatial selection. A previous study showed that location probability learning does not transfer between a letter search task and one requiring high-level decision-making, which involved guessing the location of a hidden treasure after being shown several location markers (Jiang, Swallow, Won, Cistera, \& Rosenbaum, 2015). This lack of transfer suggests a limited flexibility of probability learning, much like other forms of habit learning (Bayley et al., 2005).

\section{Conclusion}

We have shown that probability cuing can emerge with 48-96 trials of training. Additionally, we found evidence for the transfer of probability cuing between two related yet distinct tasks. Both findings are supported by behavioral and eyetracking data, and together they provide insight into viewing probability cuing as an acquired habit. Future studies on habitual attention should examine the effects of overtraining, further explore its flexibility, and evaluate the potential utility of probability cuing in inducing a transferable attentional habit between lab-administered and real-world tasks.

Author note The research reported in this publication was supported in part by NIH Grant R03 MH102586 and by an undergraduate research award from the University of Minnesota. The content is solely the responsibility of the authors and does not necessarily represent the official views of the National Institutes of Health.

\section{References}

Bayley, P. J., Frascino, J. C., \& Squire, L. R. (2005). Robust habit learning in the absence of awareness and independent of the medial temporal lobe. Nature, 436, 550-553. doi:10.1038/nature03857

Brainard, D. H. (1997). The Psychophysics Toolbox. Spatial Vision, 10, 433-436. doi:10.1163/156856897X00357
Chun, M. M., \& Jiang, Y. (1998). Contextual cueing: Implicit learning and memory of visual context guides spatial attention. Cognitive Psychology, 36, 28-71. doi:10.1006/cogp.1998.0681

Druker, M., \& Anderson, B. (2010). Spatial probability aids visual stimulus discrimination. Frontiers in Human Neuroscience, 4, 63. doi: 10.3389/fnhum.2010.00063

Geng, J. J., \& Behrmann, M. (2002). Probability cuing of target location facilitates visual search implicitly in normal participants and patients with hemispatial neglect. Psychological Science, 13, 520-525.

Graybiel, A. M. (2008). Habits, rituals, and the evaluative brain. Annual Review of Neuroscience, 31, 359-387. doi:10.1146/annurev.neuro. 29.051605.112851

Jiang, Y. V. (2017). Habitual versus goal-driven attention. Cortex (in press).

Jiang, Y. V., Swallow, K. M., Rosenbaum, G. M., \& Herzig, C. (2013). Rapid acquisition but slow extinction of an attentional bias in space. Journal of Experimental Psychology: Human Perception and Performance, 39, 87-99. doi:10.1037/a0027611

Jiang, Y. V., Swallow, K. M., Won, B.-Y., Cistera, J. D., \& Rosenbaum, G. M. (2015). Task specificity of attention training: The case of probability cuing. Attention, Perception, \& Psychophysics, 77, 50-66. doi:10.3758/s13414-014-0747-7

Jiang, Y. V., Won, B.-Y., \& Swallow, K. M. (2014). First saccadic eye movement reveals persistent attentional guidance by implicit learning. Journal of Experimental Psychology: Human Perception and Performance, 40, 1161-1173. doi:10.1037/a0035961

Jones, J. L., \& Kaschak, M. P. (2012). Global statistical learning in a visual search task. Journal of Experimental Psychology: Human Perception and Performance, 38, 152-160. doi:10.1037/a0026233

Kabata, T., \& Matsumoto, E. (2012). Cueing effects of target location probability and repetition. Vision Research, 73, 23-29. doi:10. 1016/j.visres.2012.09.014

Kristjánsson, A., \& Campana, G. (2010). Where perception meets memory: A review of repetition priming in visual search tasks. Attention, Perception, \& Psychophysics, 72, 5-18. doi:10.3758/APP.72.1.5

Kunar, M. A., Flusberg, S., Horowitz, T. S., \& Wolfe, J. M. (2007). Does contextual cuing guide the deployment of attention? Journal of Experimental Psychology: Human Perception and Performance, 33, 816-828. doi:10.1037/0096-1523.33.4.816

Pelli, D. G. (1997). The VideoToolbox software for visual psychophysics: Transforming numbers into movies. Spatial Vision, 10, 437-442. doi:10.1163/156856897X00366

Peterson, M. S., \& Kramer, A. F. (2001). Attentional guidance of the eyes by contextual information and abrupt onsets. Perception \& Psychophysics, 63, 1239-1249. doi:10.3758/BF03194537

Seger, C. A., \& Spiering, B. J. (2011). A critical review of habit learning and the basal banglia. Frontiers in Systems Neuroscience, 5, 66. doi: 10.3389/fnsys.2011.00066

Walthew, C., \& Gilchrist, I. D. (2006). Target location probability effects in visual search: An effect of sequential dependencies. Journal of Experimental Psychology: Human Perception and Performance, 32, 1294-1301. doi:10.1037/0096-1523.32.5.1294

Won, B.-Y., Lee, H. J., \& Jiang, Y. V. (2015). Statistical learning modulates the direction of the first head movement in a large-scale search task. Attention, Perception, \& Psychophysics, 77, 2229-2239. doi: 10.3758/s13414-015-0957-7

Wood, W., \& Rünger, D. (2016). Psychology of habit. Annual Review of Psychology, 67, 289-314. doi:10.1146/annurev-psych-122414033417

Yin, H. H., \& Knowlton, B. J. (2006). The role of the basal ganglia in habit formation. Nature Reviews Neuroscience, 7, 464-476. doi:10. 1038/nrn1919 